\section{Dementia}

Accepted: August 27, 2010

Published online: January 20, 2011

\title{
Diagnostic Performance of the Chinese Frontal Assessment Battery in Early Cognitive Impairment in an Asian Population
}

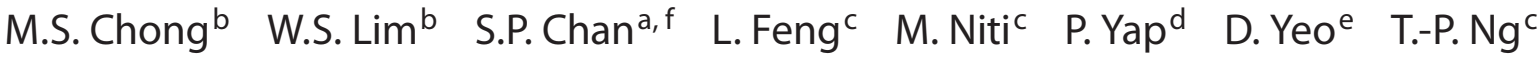 \\ ${ }^{a}$ Clinical Research Unit, ${ }^{b}$ Department of Geriatric Medicine, Tan Tock Seng Hospital, ' Gerontological Research \\ Programme, National University of Singapore, ${ }^{d}$ Department of Geriatric Medicine, Khoo Teck Puat Hospital, and \\ e Department of Neurology, Singapore General Hospital, Singapore; ${ }^{f}$ Division of Health Research, Faculty of \\ Health Sciences, La Trobe University, Melbourne, Vic., Australia
}

\section{Key Words}

Alzheimer's disease $\cdot$ Early diagnosis $\cdot$ Neuropsychology

Dementia $\cdot$ Mild cognitive impairment

\begin{abstract}
Background: The Frontal Assessment Battery (FAB) has been shown to be useful in evaluating frontal dysfunction. There is a paucity of studies validating cutoffs in the early cognitive impairment. We aim to validate the Chinese FAB in Asian subjects with mild cognitive impairment $(\mathrm{MCl})$ and early dementia. Methods: Eighty subjects with $\mathrm{MCl}$ and mild dementia and 100 cognitively healthy community subjects were studied. ROC analysis was done to determine the Chinese FAB's optimal cutoff scores for age- and education-adjusted subgroups. Results: Chinese FAB scores were significantly lower in early cognitive impairment compared with cognitively normal controls. The optimal cutoff score was 12/13 (sensitivity $92 \%$, specificity $78.7 \%$ ). A similar cutoff score was obtained following age-adjustment and for subjects with $<6$ years' education. Of note, the optimal cutoff for subjects with $\geq 6$ years' education was $13 / 14$ (sensitivity $91.8 \%$, specificity $70.3 \%$ ), an improved diagnostic performance compared to the earlier reported 11/12 cutoff. In comparison, the Mini-Mental Status Examination (MMSE) had lower rule-out accuracy (77\% sensitivity, $91.2 \%$ specificity). The combina-
\end{abstract}

tion of the Chinese FAB and MMSE was superior to either test in isolation. Conclusion: The education-adjusted Chinese FAB has good diagnostic performance, which can supplement the MMSE in early cognitive impairment evaluation with construct differences observed between the Chinese FAB and MMSE.

Copyright $\odot 2011$ S. Karger AG, Basel

\section{Introduction}

Episodic memory and executive function are the 2 cognitive domains earliest affected in mild cognitive impairment $(\mathrm{MCI})$ and very early dementia [1-4]. Previous validated screening tests in MCI in Chinese populations have focused mainly on informant-based questionnaires [5], and testing of episodic memory [6-7]. For instance, the Japanese MCI screen assesses immediate recall task, a triadic comparison task, a judgment task, a delayed free recall task, a cued-recall task and a rehearsed recall task [7]. While most of the research literature has focused on memory-biased screening instruments in this early phase of cognitive impairment, there is a paucity of literature worldwide on the specific screening tools for executive dysfunction in the earlier stages of cognitive impairment such as MCI.

\section{KARGER \\ Fax +4161306 1234 \\ E-Mail karger@karger.ch}

www.karger.com
(C) 2011 S. Karger AG, Basel

$1420-8008 / 10 / 0306-0525 \$ 26.00 / 0$

Accessible online at: www.karger.com/dem
Dr. Chong Mei Sian

Department of Geriatric Medicine, Tan Tock Seng Hospital

11 Jalan Tan Tock Seng

Singapore 308433 (Singapore)

Tel. +65 6357 7859, Fax +65 6352 4436,E-Mail Mei_Sian_Chong@ ttsh.com.sg 
The Frontal Assessment Battery (FAB) was developed by Dubois [8] for testing executive function at bedside. It is a short bedside screening instrument testing 6 subtest domains, each exploring functions related to the frontal lobe. These are namely: conceptualization, mental flexibility, motor programming, sensitivity to interference, inhibitory control and environmental autonomy. The Chinese version of the FAB was adapted for the Asian context, with the mental flexibility (verbal fluency) subtest being substituted by category fluency due to phonemic and linguistic differences in the languages [9]. The utility of the FAB as a useful test for frontal dysfunction is further confirmed by the correlations between the FAB scores and left callosomarginal and precentral segment regional blood flow on single photon emission computed tomography [10].

Most of the previous work on the FAB has focused on the differentiation of Alzheimer's disease (AD) from frontotemporal dementia [11-14], subcortical vascular dementia (VaD) [15] and (more recently) dementia with Lewy Body [16]. Existing validation studies report a range of cutoffs, either in the use of the FAB for detecting executive dysfunction in subcortical infarct (cutoff 11/12 [9]) or for differentiating $\mathrm{AD}$ from frontotemporal dementia (cutoff 11/12 [11] and 10/11 [14, 17]). In a normative population control sample, a higher cutoff of 13/14 was observed [18]. Most of these studies focused mainly on differentiating dementia etiologies and not its diagnostic performance in detection of early cognitive impairment. The utility of the FAB at an earlier stage of cognitive impairment, such as MCI, has not been studied and we postulate that a higher cutoff score, closer to the population control sample group, would be required.

In this study, we examined the diagnostic performance of the Chinese FAB compared to the Mini-Mental Status Examination (MMSE), in isolation and in combination, in the evaluation of early cognitive impairment. We also studied the influence of age- and education-cutoffs on the diagnostic performance of the Chinese FAB. Lastly, we performed subgroup analysis in the early dementia group to determine if construct differences exist between the MMSE and Chinese FAB among AD, VaD and mixed dementia.

\section{Subjects and Methods}

\section{Setting}

We included subjects with MCI and early stage $\mathrm{AD}, \mathrm{VaD}$ or mixed dementia aged $>50$ years who were seen in the Memory Clinic, Tan Tock Seng Hospital, Singapore, from November 2004 to March 2007. The cognitively normal subjects were recruited from the Singapore Longitudinal Aging Study (SLAS) arm of the Gerontology Research Programme. The recruitment of community-dwelling cognitively normal subjects instead of nondemented elderly subjects presenting to the Memory Clinic with subjective memory complaints is an important consideration for the validation part of the study. The study was submitted for ethics approval and was approved by the National Healthcare Group Domain Specific Review Board and the National University of Singapore Institutional Review Board.

\section{Evaluation}

All MCI and early AD patients underwent detailed clinical evaluation including comprehensive cognitive history from the patient and reliable informant, and a physical examination. Relevant laboratory investigations and neuroimaging (computed tomography or magnetic resonance imaging) were also performed to exclude potentially reversible causes of cognitive impairment [19]. Standardized neuropsychological assessment was performed on all MCI and early dementia subjects. The neuropsychological battery assessed verbal memory, language, visuospatial abilities and executive functioning, and consisted of word list memory (immediate recall, delayed recall and recognition), verbal fluency (animal category), the modified Boston Naming Test, the WAIS$\mathrm{R}$ subtest of block design and object assembly. Locally validated education-adjusted cutoffs have been previously established [20]. Diagnosis was subsequently made via a multidisciplinary consensus approach. The diagnosis of MCI and dementia was made independently of the Chinese FAB scores to ensure there was no circularity in the diagnostic process.

As part of the clinical assessment, the subjects were also administered cognitive screening tests and functional scales [including the Clinical Dementia Rating (CDR) scale] [21, 22]. The Chinese MMSE (CMMSE) was locally validated and modifications were made to suit the cultural context of Singapore resulting in a total possible score of 28 instead of 30 on the original MMSE [23]. Depression was assessed via the Cornell Scale for Depression in Dementia [24]. The Chinese FAB was administered to the subjects [9] during the initial visit by a single blinded rater. The Chinese FAB consists of 6 subtests: (1) conceptualization (conceptualization links between 2 objects from the same category, e.g. an orange and a banana), (2) mental flexibility (animal naming in a 1-min trial), (3) motor programming (Luria's 'fist-edge-palm' motor series), (4) sensitivity to interference (conflicting instructions in which subjects must provide an opposite response to the examiner's alternating signal, e.g. tapping once when the examiner taps twice), (5) inhibitory control (go/no-go paradigm where the subject must inhibit a response that was previously given to the same stimulus, e.g. not tapping when the examiner taps twice), and (6) environmental autonomy (placing your hands out and instructing the subject not to touch them, looking out for abnormal behavior such as imitation, utilization and prehension behavior). Each subtest is scored from $0-3$, yielding a total score of 18 .

\section{Cognitive Subgroups}

MCI was operationally defined based on the International Working Group criteria [25] as follows: (1) presence of subjective memory complaint (which should be corroborated by a reliable caregiver), (2) psychometric evidence of impairment in amnestic and nonamnestic domain $>1$ SD below the education-adjusted 
means from an earlier normative study [20], (3) relatively normal general cognitive function (CMMSE score $\geq 21$ for subjects with $\leq 6$ years' education and $\geq 24$ with $>6$ years' education [26]), (4) largely intact activities of daily living (global CDR score should not exceed 0.5) and (5) no clinical dementia (does not fulfill DSMIV criteria for dementia).

Early dementia subjects met DSM-IV criteria for dementia [27]. Their global CDR score ranged between 0.5 and 1.0, indicating the mild nature of their dementia. Subjects with dementia diagnoses other than $\mathrm{AD}, \mathrm{VaD}$ or mixed dementia were excluded. $\mathrm{AD}$ was diagnosed based upon the National Institute of Neurological and Communicative Disorders and Stroke-Alzheimer's Disease and Related Disorders Association criteria [28], while $\mathrm{VaD}$ and mixed dementia were diagnosed using National Institute of Neurological Disorders and Stroke-Association Internationale pour la Recherche et l'Enseignement en Neurosciences criteria [29]. Mixed dementia included cases who have been previously diagnosed as $\mathrm{AD}$, but who now present with a clinically relevant stroke resulting in further decline of cognitive status, or cases who otherwise fulfill criteria for $\mathrm{VaD}$ but where it is adjudged that there is a very high clinical suspicion of concomitant AD. The operational definition of these etiologic criteria in our Memory Clinic has been described in detail elsewhere [30].

Healthy elderly controls were obtained from the SLAS arm of the Gerontology Research Programme, a community-based prospective cohort study of aging and health. The methodology has been described in detail elsewhere [31-33]. In brief, all residents (Singapore citizens and permanent residents) of the southeast region of Singapore who were aged $\geq 55$ years were identified from a door-to-door census and invited to participate in the study. Out of a total of 2,808 participants who enrolled in the baseline examination from September 2003 to December 2005, 167 subjects had the Chinese version of the FAB administered. To ensure the purity of the controls in the study, we employed the following locally validated discriminatory criteria in the selection of our control group: nondemented, Informant Questionnaire on Cognitive Decline in the Elderly <3.4 [34], normal MMSE (MMSE $\geq 26$ ), CDR of 0 and not depressed (15-item Geriatric Depression Scale $<4)[24,35,36]$. Investigators of the SLAS study had previously reported that a cutoff of 25/26 on the 30 -item modified MMSE was able to differentiate cognitive impairment from healthy controls.

\section{Statistical Analysis}

We obtained demographic data (sex, years of education and age) and scores on bedside screening instruments (CMMSE, Chinese FAB total and subscores, CDR global and sum of boxes scores). Statistical analysis was performed on SPSS 17.0 statistical software. $p<0.05$ was determined to be statistically significant.

Demographic and Chinese FAB total and subscore differences between those with and without early cognitive impairment was examined using a Mann-Whitney U test. We also compared the age and education-adjusted Chinese FAB total and subscore differences between normal, MCI and early dementia subjects. The optimal cutoff scores to determine the FAB to identify cognitive impairment were determined by the receiver operator characteristic (ROC) technique, which was used to determine the Chinese FAB's optimal cutoff score for the entire cohort (unadjusted) and following age adjustments (age $<75, \geq 75$ years) and educationadjustments ( $\leq 6$ years, $>6$ years). The choice of age-adjustments was made based on those used for previous local validation work [20].

To allow for comparison of MMSE scores between the SLAS and memory clinic cohorts, we removed 2 subitems (time and area of Singapore) from the modified MMSE of the SLAS cohort to obtain a total score of 28. Items in both the modified MMSE and CMMSE are identical after this adjustment, except for the 3 -item recall (ball, flag and tree in the modified MMSE vs. lemon, key and balloon in CMMSE). We judged these items to assess the same domain of delayed recall and to be of comparable difficulty, thus of sufficient test equivalence to allow for comparison between the 2 cohorts. We looked at the diagnostic test performance of the Chinese FAB and MMSE, in combination and in isolation, for those with and without early cognitive impairment.

Finally, we performed subgroup analysis on the early dementia subgroups of $\mathrm{AD}, \mathrm{VaD}$ and mixed dementia to examine the construct differences between the Chinese FAB and MMSE using a Kruskal-Wallis test and Spearman's correlation for nonparametric tests.

\section{Results}

Eighty subjects with early cognitive impairment (MCI and mild dementia) and 100 cognitively intact subjects were studied. Of the 80 early cognitive impairment subjects, we included $21 \mathrm{MCI}$ subjects and 59 early dementia subjects. The MCI group comprised the following: $2 \mathrm{am}$ nestic single-domain, 5 amnestic multidomain, 10 nonamnestic single domain and 4 nonamnestic multidomain MCI.

\section{Comparison of Cognitively Normal and Early \\ Cognitive Impairment Subjects}

There were significant differences in age and education between those with cognitive impairment (MCI and mild dementia) and cognitively intact elderly (table 1). Significance differences in adjusted MMSE scores were noted between the groups. The mean CDR global score was $0.5 \pm 0.1$ and $0.94 \pm 0.2$ with a CDR sum of boxes score of $1.6 \pm 0.9$ and $3.9 \pm 1.6$ for MCI and early dementia subjects, respectively, indicating the early nature of cognitive impairment in the early dementia subjects. The subjects did not have significant depressive symptoms on the Cornell Scale for Depression in Dementia.

Significant differences were also seen in the Chinese FAB total score as well as the Chinese FAB subscore items. The mean total Chinese FAB in the cognitively intact group was $15.96 \pm 2.61$ and $9.74 \pm 3.5$ in the early cognitive impairment (MCI and early dementia) group ( $\mathrm{p}<$ 0.05). The Chinese FAB had good convergent validity with the decreasing total scores and subscores observed from cognitively normal to early dementia subjects, with 
Table 1a. Demographics of cognitive subgroups

\begin{tabular}{lcc}
\hline & $\begin{array}{l}\text { Normal } \\
(\mathrm{n}=100)\end{array}$ & $\begin{array}{l}\text { Early cognitive } \\
\text { impairment }(\mathrm{n}=80)\end{array}$ \\
\hline $\begin{array}{l}\text { Demographics } \\
\text { Sex (M:F) }\end{array}$ & $50: 63$ & $40: 40$ \\
Age, years & $67.3 \pm 8.15$ & $71.7 \pm 7.77^{*}$ \\
Education, years & $9.6 \pm 4.4$ & $6.6 \pm 4.6^{*}$ \\
\hline Cognitive screening instruments & & \\
Adjusted MMSE score & $26.7 \pm 1.2$ & $20.3 \pm 4.5^{*}$ \\
FAB & & \\
$\quad$ Conceptualization & $2.29 \pm 0.81$ & $0.89 \pm 1.1^{*}$ \\
$\quad$ Mental flexibility & $2.85 \pm 0.39$ & $1.78 \pm 0.75^{*}$ \\
$\quad$ Motor programming & $2.85 \pm 0.39$ & $1.91 \pm 1.07^{*}$ \\
Sensitivity to interference & $2.75 \pm 0.67$ & $1.41 \pm 1.27^{*}$ \\
Inhibitory control & $2.23 \pm 1.03$ & $1.1 \pm 1.18^{*}$ \\
Environmental autonomy & $2.99 \pm 0.10$ & $2.7 \pm 0.85^{*}$ \\
$\quad$ FAB total score & $15.96 \pm 2.06$ & $9.74 \pm 3.5^{*}$ \\
\hline
\end{tabular}

Table 1b. Analysis by cognitive subgroups (normal, MCI and early dementia subjects)

\begin{tabular}{lrrr}
\hline & Normal $(\mathrm{n}=100)$ & MCI $(\mathrm{n}=21)$ & Dementia $(\mathrm{n}=59)$ \\
\hline $\begin{array}{l}\text { Demographics } \\
\text { Age, years }\end{array}$ & $63.7 \pm 6.51$ & $69.3 \pm 7.91$ & $72.7 \pm 7.59^{*}$ \\
Education, years & $9.6 \pm 4.39$ & $7.1 \pm 4.40$ & $6.5 \pm 4.09^{*}$ \\
\hline Cognitive screening instruments & & & \\
Adjusted MMSE score & $26.6 \pm 1.2$ & $23.5 \pm 4.12$ & $19.36 \pm 4.35^{*}$ \\
FAB & & & \\
$\quad$ Conceptualization & $2.29 \pm 0.81$ & $1.48 \pm 1.29$ & $0.68 \pm 0.94^{*, a}$ \\
$\quad$ Mental flexibility & $2.85 \pm 0.39$ & $1.81 \pm 0.68$ & $1.76 \pm 0.77^{*, a}$ \\
$\quad$ Motor programming & $2.85 \pm 0.39$ & $2.14 \pm 1.06$ & $1.83 \pm 1.07^{*, a}$ \\
Sensitivity to interference & $2.75 \pm 0.67$ & $1.62 \pm 1.20$ & $1.34 \pm 1.29^{*, a}$ \\
Inhibitory control & $2.23 \pm 1.03$ & $1.52 \pm 1.21$ & $0.88 \pm 1.13^{*, a}$ \\
$\quad$ Environmental autonomy & $2.99 \pm 0.10$ & $2.67 \pm 0.91$ & $2.71 \pm 0.83^{*, a}$ \\
FAB total score & $15.96 \pm 2.06$ & $11.24 \pm 3.63$ & $9.20 \pm 3.31^{*, a}$ \\
\hline
\end{tabular}

Values denote means \pm SD unless specified otherwise.

${ }^{*} \mathrm{p}<0.05$. ${ }^{\mathrm{a}}$ Differences in FAB remained after age- and education-adjustment.

MCI subjects having intermediate scores $(\mathrm{p}<0.05)$. These differences in the cognitive subgroups remained after adjustments for age and education.

\section{Cutoff and Diagnostic Performance}

Using ROC analysis, the optimal cut-off score was 12/13 for the whole group [sensitivity (Sn): $92 \%$, specificity (Sp): $78.7 \%$ ] with classification accuracy (Ac) of $86.1 \%$. A similar cutoff score was obtained following age-adjustment ( $<75$ years and $\geq 75$ years). However, for subjects with $<6$ years' education, the cutoff was $12 / 13$ (Sn: $77.8 \%$, Sp: $95.2 \%$, Ac: $88.4 \%$ ) and increased to $13 / 14$ with $\geq 6$ years' education (Sn: 91.8\%, Sp: 70.3\%, Ac: $84.5 \%)$. In comparison, the diagnostic performance of the MMSE showed lower rule-out accuracy (Sn: 77\%, Sp: 91.2\%, Ac: 83.3\%). The combination of MMSE and education-adjusted Chinese FAB performance failure was superior (Sn: $91.3 \%$, Sp: 77\%, Ac: 88.9\%) compared to either test in isolation (table 2).

\section{Subgroup Analysis of Early Dementia Subjects}

The early dementia subjects consisted of $31 \mathrm{AD}, 12 \mathrm{VD}$ and 16 mixed dementia subjects. They all had good motor power $\geq 4$. No significant age or education differences 
Table 2. Diagnostic performance of the Chinese FAB and adjusted MMSE scores

\begin{tabular}{|c|c|c|c|c|c|}
\hline Cognitive test & Cutoff & AUC (95\% CI) & $\begin{array}{l}\text { Sensitivity } \\
\%\end{array}$ & $\begin{array}{l}\text { Specificity } \\
\%\end{array}$ & $\begin{array}{l}\text { Diagnostic } \\
\text { accuracy, \% }\end{array}$ \\
\hline \multicolumn{6}{|l|}{ Chinese FAB } \\
\hline Chinese FAB, unadjusted & $12 / 13$ & $0.93(0.89-0.97)$ & 92 & 78.7 & 86.1 \\
\hline \multicolumn{6}{|l|}{ Chinese FAB, age-adjusted } \\
\hline$<75$ years & $12 / 13$ & $0.93(0.86-0.98)$ & 92.6 & 76.5 & 86.9 \\
\hline$\geq 75$ years & $12 / 13$ & $0.84(0.69-0.99)$ & 83.3 & 81.8 & 82.6 \\
\hline \multicolumn{6}{|l|}{ Chinese FAB, education-adjusted } \\
\hline$<6$ years & $12 / 13$ & $0.94(0.89-0.99)$ & 77.8 & 95.2 & 88.4 \\
\hline$\geq 6$ years & $13 / 14$ & $0.91(0.85-0.97)$ & 91.8 & 70.3 & 84.5 \\
\hline Adjusted MMSE & $25 / 26$ & $0.94(0.90-0.98)$ & 77 & 91.2 & 83.3 \\
\hline \multicolumn{6}{|l|}{ Combined education-adjusted } \\
\hline Chinese FAB and MMSE & & & 91.3 & 77 & 88.9 \\
\hline
\end{tabular}

Table 3. Subgroup analysis of the different dementia etiologic subgroups in early dementia subjects $(\mathrm{n}=59)$

\begin{tabular}{lccc}
\hline & AD $(\mathrm{n}=31)$ & $\mathrm{VD}(\mathrm{n}=12)$ & Mixed $(\mathrm{n}=16)$ \\
\hline Demographics & & & \\
Sex (M:F) & $15: 16$ & $7: 5$ & $10: 6$ \\
Age, years & $71.7 \pm 8.5$ & $72.5 \pm 6.56$ & $74.5 \pm 6.5$ \\
Education, years & $7.4 \pm 4.9$ & $0.8 \pm 4.2$ & $6.1 \pm 4.5$ \\
CDR (global) & $0.94 \pm 0.17$ & $3.70 \pm 1.27$ & $0.97 \pm 0.13$ \\
CDR (SOB) & $4.0 \pm 1.9$ & & $3.9 \pm 1.36$ \\
\hline Cognitive screening instruments & & $18.83 \pm 3.6$ & \\
MMSE (/28) & $18.8 \pm 4.6$ & & $20.8 \pm 4.3$ \\
FAB & & & \\
$\quad$ Conceptualization & $0.77 \pm 1.02$ & $1.58 \pm 0.90$ & $0.56 \pm 0.81$ \\
$\quad$ Mental flexibility & $1.77 \pm 0.72$ & $1.58 \pm 1.08$ & $1.88 \pm 0.89$ \\
$\quad$ Motor programming & $1.90 \pm 1.04$ & $0.5 \pm 0.90^{*}$ & $1.88 \pm 1.15$ \\
$\quad$ Sensitivity to interference & $1.65 \pm 1.25^{*}$ & $0.25 \pm 0.62^{*}$ & $1.31 \pm 1.40^{*}$ \\
$\quad$ Inhibitory control & $0.90 \pm 1.16^{*}$ & $2.1 \pm 1.38^{*}$ & $2.81 \pm 0.75^{*}$ \\
$\quad$ Environmental autonomy & $2.9 \pm 0.40^{*}$ & $6.6 \pm 2.19^{*}$ & $9.8 \pm 3.4^{*}$ \\
$\quad$ FAB total score & $9.9 \pm 3.19^{*}$ & &
\end{tabular}

Values denote means \pm SD unless specified otherwise.

${ }^{*} \mathrm{p}<0.05 . \mathrm{SOB}=$ Sum of boxes.

were observed. No significant differences were observed in CMMSE scores although the CMMSE score was higher in the mixed dementia group (table 3). Statistical differences were observed in the Chinese FAB total scores with $\mathrm{VaD}$ scoring lowest. Significant differences were seen in the sensitivity in interference, inhibitory control and environmental autonomy in the Chinese FAB subscores.
Spearman's correlation analysis revealed the Chinese FAB and neuropsychological delayed memory score to be weakly correlated in MCI and early dementia subjects $(\mathrm{r}=0.25, \mathrm{p}=0.3$ and $\mathrm{r}=0.02, \mathrm{p}=0.9$, respectively). Among the different dementia etiological subgroups, there was weak correlation of the Chinese FAB and MMSE in AD $(\mathrm{r}=0.01, \mathrm{p}=0.94)$ and $\mathrm{VaD}(\mathrm{r}=0.02, \mathrm{p}=0.95)$, in contrast to the moderate correlation for the mixed dementia subgroup $(\mathrm{r}=0.41, \mathrm{p}=0.11)$. 


\section{Discussion}

The key findings from this study include the use of education-adjusted Chinese FAB scores to effectively diagnose early cognitive impairment, namely MCI and early dementia, in elderly Asian subjects. Higher cutoff scores of 13/14 are required for those with higher education ( $\geq 6$ years). We also demonstrated the improved diagnostic performance of a combination of the memorybased MMSE and Chinese FAB in the diagnosis of early cognitive impairment. This study showed construct differences between the MMSE and Chinese FAB in the different etiologic subgroups, which supports the complementary use of the Chinese FAB in early cognitive evaluation to supplement memory-biased cognitive tools such as the MMSE.

The optimal cutoff Chinese FAB score (whole group) of $12 / 13$ together with $13 / 14$ in those with $\geq 6$ years' education with good accuracy compared favorably with earlier validation studies. Existing validation studies observed cutoffs of 11/12 for executive dysfunction in subcortical infarct (Sn: 69.2\%, Sp: 87.7\%, Ac: 80.6\%) [9] and differentiated AD (cutoff 11/12; Sn: 77\%, Sp: 87\%) [2] from frontotemporal dementia (cutoff 10/11; Sn: 85-92\%, Sp: 85\%) [8]. The cutoff scores in our study are more similar to the normative values in an Italian population control sample [15]. This is likely related to the fact that we included subjects in the earlier stages of cognitive impairment with CDR scores $\leq 1$ [37] and the use of community-dwelling healthy elderly controls who met the operational definition of cognitively normal (in contrast to including subjects presenting to the specialist memory clinic with subjective memory complaints who were subsequently diagnosed as normal following evaluation).

The novel finding of superior diagnostic performance of the combination of memory-based (MMSE) and executive-function (Chinese FAB) testing compared to either test in isolation supports the value of the Chinese FAB being a supplementary test to the widely-used MMSE when evaluating cognitive impairment. There is currently a dearth of literature on combined memory and executive function bedside testing in early cognitive impairment, despite a recent longitudinal study showing executive function decline to precede memory decline by 3 years, followed subsequently by tandem memory and executive function decline [38]. The above temporal sequence of cognitive domain involvement supports our study findings of the improved performance of combination tests. The FAB is a relatively brief and easy cognitive test, requiring about $5 \mathrm{~min}$ to administer. This enhances its practicality in the busy clinic setting. In contrast, other executive function screening tests such as the 25-item EXIT 25 [39] takes a significantly longer time for administration and may require a higher literacy level. Another commonly described test, the clock drawing test, requires the subject to have sufficient pen-holding skills, which can be challenging in the local elderly, a significant proportion of which are illiterate and have not received any formal education.

The demonstration of construct differences in the Chinese FAB and MMSE is supported by the subgroup analysis in the etiologic subgroups demonstrating poor correlation of the Chinese FAB with delayed memory, and differential scores in the Chinese FAB and MMSE despite a similar CDR global and sum of boxes score (table 3). This is in line with existing literature that executive function deficit may be more prominent in early $\mathrm{VaD}[40$, 41] and contributes most to cognitive and functional impairment in $\mathrm{VaD}$. In mixed dementia, where both executive function and episodic memory are affected, the superior correlation compared with $\mathrm{AD}$ and $\mathrm{VaD}$ further supports the differential psychometric property of the Chinese FAB compared to the memory-biased MMSE. This supports the construct validity of the Chinese FAB being a measure of executive function. Therefore, the above findings also explain the superior performance of combination testing with the MMSE and Chinese FAB compared to either test alone.

The strength of the study lies in the inclusion of subjects in the earlier stages of cognitive impairment (namely MCI and mild dementia), compared to previous validation studies where the subjects had already well-established dementia. This has implications on clinical practice as it permits the use of this bedside executive function instrument as a screen in patients suspected of having cognitive impairment with subtle cognitive changes on presentation. The main thrust of the paper is to examine the utility of the FAB as a screening tool for detection of early cognitive impairment regardless of specific etiology. The merit of our approach is that it reproduces the day-to-day clinical scenario where a screening tool would be used to detect early cognitive impairment in a population that is undifferentiated for etiology. This approach would confer more relevance and 'face validity' than previous papers that restricted the assessment of diagnostic performance to specific etiological causes.

The limitations of the study include the under-representation of the small group of old-old subjects more than 75 years of age, thus not permitting the extrapolation of study findings to this age population. The overall sample 
size of the early cognitive impairment cohort also does not permit separate detailed analysis of the MCI subjects and the MCI subtypes. The combination of 2 different study cohorts posed certain challenges; however, rigorous selection of controls to avoid 'contamination' and sufficient test equivalence between the CMMSE and adjusted MMSE allowed for cross-comparisons between the 2 study cohorts. Another potential criticism of the paper would be whether the predominance of nonamnestic MCI subjects contributed to the strong performance of the FAB compared to the MMSE. We compared the MMSE, FAB subscores and total scores and found neither demonstrable trends nor significant differences in the MCI subtypes to suggest this.

In conclusion, our study supports the viability of using the education-adjusted FAB as a screening instrument in detection of early cognitive impairment in elderly subjects using a cutoff of $12 / 13$ ( $<6$ years' education) and $13 / 14$ ( $\geq 6$ years' education). Optimal diagnostic perfor- mance was obtained by the FAB supplementation to the MMSE, a strategy which was supported by the demonstration of construct differences between the Chinese FAB and MMSE.

\section{Acknowledgements}

The authors would like to thank the team members of the Cognition and Memory Disorders Service, Tan Tock Seng Hospital, for their help and clinical contribution to the study.

\section{Disclosure Statement}

The Singapore Longitudinal Aging Study (SLAS) arm of the study was supported by a research grant (No. 03/1/21/17/214) from the Biomedical Research Council, Agency for Science, Technology and Research (ASTAR).

\section{References}

1 Grober E, Hall CB, Lipton RB, et al: Memory impairment, executive dysfunction and intellectual decline in preclinical Alzheimer disease. J Int Neuropsychol Soc 2008; 14 : 266-278.

2 Chen P, Ratcliff G, Belle SH, et al: Cognitive tests that discriminate between presymptomatic $\mathrm{AD}$ and those who remain no dementia. Neurology 2000;55:1847-1853.

-3 Twamley EW, Legendre Ropacki SA, Bondi MW: Neuropsychological and neuroimaging changes in preclinical Alzheimer disease. J Int Neuropsychol Soc 2006;12:707735.

$\checkmark 4$ Lam LCW, Lui VWC, Chiu HFK, Chan SSM, Tam CWC: Executive function impairment in community elderly subjects with questionable dementia. Dement Geriatr Cogn Disord 2005; 19:86-90.

$\checkmark 5$ Li M, Ng TP, Kua EH, Ko SM: Brief informant screening test for mild cognitive impairment and early Alzheimer's disease. Dement Geriatr Cogn Disord 2006;21:392-402.

-6 Lam LC, Tam CW, Lui VW, Chan WC, Chan SS, Chiu HF, Leung T, Tham MK, Ho KS, Chan WM: Screening of mild cognitive impairment in Chinese older adults - a multistage validation of the Chinese abbreviated mild cognitive impairment test. Neuroepidemiology 2008;30:6-12.

-7 Cho A, Sugimura M, Nakano S, Yamada T: The Japanese MCI screen for early detection of Alzheimer's disease and related disorders. Am J Alzheimers Dis Other Demen 2008;23: 162-166.
8 Dubois B, Slachesvsky A, Litvan I, et al: The FAB. A Frontal Assessment Battery at bedside. Neurology 2000;55:1621-1626.

-9 Mok VCT, Wong A, Yim P, et al: The validity and reliability of Chinese Frontal Assessment Battery in evaluating executive dysfunction among Chinese patients with small subcortical infarct. Alz Dis Assoc Disord 2004; 18:68-74.

10 Yoshida H, Terada S, Sato S, et al: Frontal Assessment Battery and brain perfusion imaging in early dementia. Dement Geriatr Cogn Disord 2009;27:133-138.

-11 Slachevsky A Villalpando JM, Sarazin M, Hanhn-Barma V, Pillon B, Dubois B: Frontal Assessment Battery and differential diagnosis of frontotemporal dementia and Alzheimer disease. Arch Neurol 2004;61:11041107.

12 Perri R, Koch G, Carlesimo GA, et al: Alzheimer's disease and frontal variant of frontotemporal dementia. A very brief battery for cognitive and behavioural distinction. J Neurol 2005;252:1238-1244.

13 Lipton AM, Ohman KA, Womack KV, Hynan LS, Ninman ET, Lacritz LH: Subscores of the FAB differentiate frontotemporal lobar degeneration from AD. Neurology 2005:65:726-731.

14 Nakaaki S, Murata Y, Sato J, et al: Reliability and validity of the Japanese version of the Frontal Assessment Battery in patients with the frontal variant of frontotemporal dementia. Psy Clin Sci 2007;61:78-83.
15 Oguro H, Yamaguchi S, Abe S, et al: Differentiating Alzheimer's disease from subcortical vascular dementia with FAB test. J Neurol 2006;253:1490-1494.

16 Hanyu H, Sato T, Kume K, et al: Differentiation of dementia with Lewy bodies from Alzheimer disease using the Frontal Assessment Battery. Int J Geriatr Psy 2009;24: 1034-1035.

17 Iavarone A, Ronga B, Pellegrino L, et al: The Frontal Assessment Battery (FAB): normative data from an Italian sample and performances of patients with Alzheimer's disease and frontotemporal dementia. Funct Neurol 2004;19:191-195.

18 Appollonio I, Leone M, Isella V, et al: The Frontal Assessment Battery (FAB): normative values in an Italian population sample. Neurol Sci 2005;26:108-116.

19 Chong MS, Sahadevan S: An evidence-based clinical approach to the diagnosis of dementia. Ann Acad Med Singapore 2003;32:740748

20 Sahadevan S, Lim JP, Tan NJ, Chan SP: Psychometric identification of early Alzheimer disease in an elderly Chinese population with differing educational levels. Alzheimer Dis Assoc Disord 2002;16:65-72.

21 Morris JC: The Clinical Dementia Rating (CDR): current version and scoring rules. Neurology 1993;43:2412-2414.

22 WS Lim, JJ Chin, CK Lam, PPJ Lim, Sahadevan S: Clinical Dementia Rating experience of a multi-racial Asian population. Alzheimer Dis Assoc Disord 2005;19:135-142. 
23 Sahadevan S, Tan NJ, Tan T, Tan S: Cognitive testing of elderly Chinese people in Singapore: influence of education and age on normative scores. Age Ageing 1997;26:481-486.

24 Lam CK, Lim PP, Low BL, Ng LL, Chiam PC, Sahadevan S: Depression in dementia: a comparative and validation study of four brief scales in the elderly Chinese. Int J Geriatr Psychiatry 2004;19:422-428.

25 Peterson RC: Mild cognitive impairment as a diagnostic entity. J Int Med 2004;256:183194.

26 Sahadevan S, Lim PP, Tan NJ, Chan SP: Diagnostic performance of two mental status tests in the older Chinese: influence of education and age on cut-off values. Int J Geriatr Psychiatry 2000;15:234-241.

27 Dementia. American Psychiatric Association, Committee on Nomenclature and statistics. Diagnostic and Statistical Manual of Mental Disorders, ed 4, revised. Washington, American Psychiatric Association.

28 McKhann G, Drachman D, Folstein M, Katzman R, Price D, Stadlan EM: Clinical diagnosis of Alzheimer's disease: report of the NINCDS-ADRDA Work Group under the auspices of Department of Health and $\mathrm{Hu}$ man Services Task Force on Alzheimer's disease. Neurology 1984;34:939-944.
29 Roman GC, Tatemichi TK, Erkinjuntti T, et al: Vascular dementia: diagnostic criteria for research studies. Report of the NINDSAIREN International Workshop. Neurology 1993;43:250-260.

30 Chan M, Lim WS, Sahadevan S: Stage-independent and stage-specific phenotypic differences between vascular dementia and Alzheimer's disease. Dement Geriatr Cogn Disord 2008;26:513-521.

31 Yap PL, Ng TP, Niti M, Yeo D, Henderson L: Diagnostic performance of clock drawing test by CLOX in an Asian Chinese population. Dement Geriatr Cogn Disord 2007;24: 193-200.

32 Ng TP, Niti M, Feng L, Kua EH, Yap KB: Albumin, apolipoprotein E-epsilon4 and cognitive decline in community-dwelling Chinese older adults. J Am Geriatr Soc 2009;57: 101-106.

33 Ng TP, Feng L, Niti M, Kua EH, Yap KB: Folate, vitamin B12, homocysteine, and depressive symptoms in a population sample of older Chinese adults. J Am Geriatr Soc 2009;57: 871-876.

34 Lim HJ, Lim JP, Anthony P, Yeo DH, Sahadevan $S$ : Prevalence of cognitive impairment amongst Singapore's elderly Chinese: a community-based study using the ECAQ and the IQCODE. Int J Geriatr Psychiatry 2003;18: 142-148.

35 Yesavege J: The use of rating depression series in the elderly; in Poon LW (ed): Clinical Memory Assessment of Older Adults. Washington, American Psychological Association, 1986.
36 Broekman BF, Nyunt SZ, Niti M, Jin AZ, Ko SM, Kumar R, Fones CS, Ng TP: Differential item functioning of the Geriatric Depression Scale in an Asian population. J Affect Disord 2008;108:285-290.

37 Kugo A: Japanese version of the Frontal Assessment Battery for dementia. Psychiatry Res 2007;153:69-75.

38 Carlson MC, Xue QL, Zhou J, Fried LP: Executive decline and dysfunction precedes declines in memory: the Women's Health and Aging Study II. Gerontol A Biol Sci Med Sci 2009;64:110-117.

39 Royall DR, Mahurin RK, Gray KF: Bedside assessment of executive cognitive impairment: the executive interview. J Am Geriatr Soc 1992;40:1221-1226.

40 Traykov L, Baudic S, Thibaudet MC, Rigaud AS, Smagghe A, Boller F: Neuropsychological deficit in early subcortical vascular dementia: comparison to Alzheimer's disease. Dement Geriatr Cogn Disord 2002;14:2632.

41 Padovani A, Di Piero V, Bragoni M, Iacoboni M, Gualdi GF, Lenzi GL: Patterns of neuropsychological impairment in mild dementia: a comparison between Alzheimer's disease and multi-infarct dementia. Acta Neurol Scand 1995;92:433-442. 